\title{
Overexpression of collagen type III in injured myocardium prevents cardiac systolic dysfunction by changing the balance of collagen distribution
}

Ayako Uchinaka, PhD, ${ }^{a}$ Maho Yoshida, MSc, ${ }^{b}$ Kiyoka Tanaka, MSc, ${ }^{b}$ Yoshinosuke Hamada, PhD, Seiji Mori, PhD, ${ }^{\mathrm{c}, \mathrm{d}}$ Yoshitaka Maeno, PhD, ${ }^{\mathrm{e}}$ Shigeru Miyagawa, MD, PhD, ${ }^{\mathrm{f}}$ Yoshiki Sawa, MD, PhD, Kohzo Nagata, MD, PhD, ${ }^{a}$ Hirofumi Yamamoto, $\mathrm{MD}, \mathrm{PhD},{ }^{\mathrm{c}}$ and Naomasa Kawaguchi, $\mathrm{PhD}^{\mathrm{b}, \mathrm{g}}$

\section{ABSTRACT}

Objective: Left ventricular (LV) remodeling alters the contractile and relaxation properties and induces myocardial stiffness. As LV remodeling progresses, the amount of collagen type III (Col3) is gradually decreased, being replaced by collagen type I (Col1). We evaluated whether Col3 overexpression improved cardiac function and remodeling in a rat with ischemic cardiomyopathy (ICM). We also investigated the functional motif and mechanism of thrombin-cleaved $\mathrm{N}$-terminal osteopontin (N-OPN) on cardiac remodeling.

Methods: The rats with ICM were divided into 3 groups: ligation only (Control) group and groups transplanted with nontransfected fibroblast sheets (normal $\mathrm{Fb}$ group) or with $\mathrm{Col} 3$-secretory fibroblast sheets ( $\mathrm{Col} 3 \mathrm{Fb}$ group). A gelatin hydrogel containing the N-terminal fragment (N-OPN), N-OPN lacking the SVVYGLR sequence $(\triangle \mathrm{SV})$, the Arg-Gly-Asp (RGD) sequence ( $\triangle \mathrm{RGD})$, RGD and SVVYGLR sequences ( $\triangle \mathrm{RGD}-\mathrm{SV}$ ), SVVYGLR alone (SV), or a random SV peptide was implanted into an ICM model rat.

Results: The Col3 $\mathrm{Fb}$ group exhibited significantly attenuated LV systolic dysfunction. LV dilatation, myocyte hypertrophy, and LV fibrosis at the infarcted area were also attenuated by $\mathrm{Col} 3 \mathrm{Fb}$ implantation. Furthermore, N-OPN, $\triangle \mathrm{RGD}$, and SV peptide suppressed the depression of cardiac function, LV dilatation, and myocyte hypertrophy, and also induced increased $\mathrm{Col} 3$ expression and reduction in the ratio of Coll to $\mathrm{Col} 3$ in the infarcted and border areas.

Conclusions: Overexpression of $\mathrm{Col} 3$ improved cardiac function by changing the balance of collagen distribution in LV remodeling. The SVVYGLR motif of the thrombin-cleaved N-OPN and SV peptide attenuated cardiac dysfunction by increasing $\mathrm{Col} 3$ and changing the pattern of collagen balance in the impaired area. (J Thorac Cardiovasc Surg 2018;156:217-26)

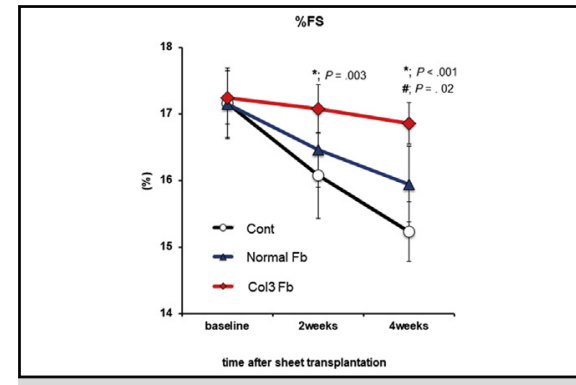

Cardiac systolic function after implantation of Col3secretory cell sheets.

Central Message

Increased collagen type III expression in injured myocardium maintains cardiac systolic function.

Perspective

$\mathrm{Col} 3$ is the collagen fiber laid down in cardiac injury. It is then replaced by Coll, which consequently leads to increased stiffness of the left ventricular (LV) wall and LV systolic dysfunction. We found that increasing $\mathrm{Col} 3$ distribution attenuated cardiac systolic dysfunction by changing the balance of the collagen ratio in LV remodeling. This finding provides a novel strategy for cardiac therapeutics.

See Editorial Commentary page 227
Following myocardial infarction (MI), cardiomyocytes are triggered to undergo apoptosis, with the apoptotic region being replaced by collagen fiber along with a gradual loss of elastic fiber. The fibrotic left ventricular (LV) wall is intolerant of intracardiac pressure; consequently, LV

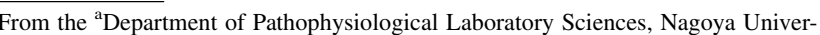
sity Graduate School of Medicine, Aichi, Japan; 'bivision of Health Sciences, Department of Cardiovascular Pathology, ${ }^{\mathrm{c}}$ Division of Health Sciences, Department of Molecular Pathology, and Departments of ${ }^{\mathrm{f}}$ Cardiovascular Surgery and ${ }^{g}$ Drug Discovery Cardiovascular Regeneration, Osaka University Graduate School of Medicine, Osaka, Japan; ${ }^{\mathrm{d}}$ Department of Medical Technology, Faculty of Health Sciences, Morinomiya University of Medical Sciences, Osaka, Japan; and ${ }^{\mathrm{e}}$ Department of Food and Nutritional Sciences, Collage of Bioscience and Biotechnology, Chubu University, Aichi, Japan.
}

remodeling, such as expansion of the infarcted area, ventricular dilatation, myocyte hypertrophy, and thinning of the ventricular wall, ensues. ${ }^{1-3}$ Extracellular matrix (ECM) deposition and organization play major roles in LV remodeling, with progressive LV remodeling

Received for publication Aug 20, 2017; revisions received Jan 9, 2018; accepted for publication Jan 11, 2018; available ahead of print March 15, 2018.

Address for reprints: Naomasa Kawaguchi, PhD, Department of Drug Discovery Cardiovascular Regeneration, Osaka University Graduate School of Medicine, 2-2 Yamada-oka, Suita, Osaka 565-0871, Japan (E-mail: kawaguch@sahs.med. osaka-u.ac.jp).

$0022-5223 / \$ 36.00$

Copyright (C) 2018 by The American Association for Thoracic Surgery

https://doi.org/10.1016/j.jtcvs.2018.01.097 


$$
\begin{aligned}
& \text { Abbreviations and Acronyms } \\
& \begin{aligned}
\text { Col1 } & =\text { collagen type I } \\
\text { Col3 } & =\text { collagen type III } \\
\text { COL3a1 } & =\text { collagen type III } \alpha 1 \text { chain } \\
\text { ECM } & =\text { extracellular matrix } \\
\mathrm{EF} & =\text { ejection fraction } \\
\text { Fb } & =\text { fibroblast } \\
\text { FS } & =\text { fractional shortening } \\
\text { ICM } & =\text { ischemic cardiomyopathy } \\
\text { LV } & =\text { left ventricular } \\
\text { MI } & =\text { myocardial infarction } \\
\text { N-OPN } & =\text { N-terminal osteopontin } \\
\text { OPN } & =\text { osteopontin } \\
\text { RGD } & =\text { Arg-Gly-Asp } \\
\text { SMA } & =\text { smooth muscle actin }
\end{aligned}
\end{aligned}
$$

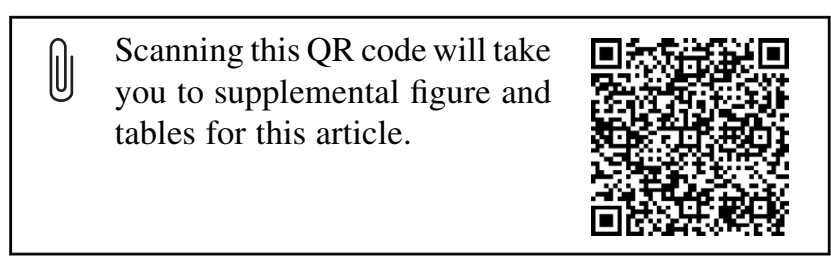

associated with increased interstitial collagen fiber a major cause of diastolic heart failure after MI. ${ }^{4-6}$ Notably, the ratio of collagen type I (Col1) and $\mathrm{Col} 3$ within cardiac interstitial tissue has an influence on both cardiac diastolic and systolic function. In the LV remodeling process, the level of Col1 is increased whereas that of $\mathrm{Col} 3$ is decreased. ${ }^{4,7,8}$ In particular, this increase in the ratio of Col1 to $\mathrm{Col} 3$ contributes to reduced elasticity of the LV wall and LV dilation, ${ }^{9,10}$ because Col3, whose helical molecules form a homotrimer of $\alpha 1$ chains, ${ }^{11}$ is an important component for maintaining the normal morphological structure and physiological function of cardiac tissue. ${ }^{4}$

Osteopontin (OPN) is an acidic phosphorylated glycoprotein containing an integrin-binding Arg-Gly-Asp (RGD) sequence. ${ }^{12}$ OPN biological functions, such as bone remodeling, macrophage response, cell migration, and adhesion, are modulated by thrombin cleavage generating $\mathrm{N}$ - and $\mathrm{C}$-terminal fragments. ${ }^{13-15}$ Thrombin cleavage of OPN is required for integrin $\alpha_{9} \beta_{1}$ interaction with the resulting $\mathrm{N}$-terminal fragment via the motif ${ }^{162}$ SVVYGLR ${ }^{168} \cdot{ }^{16,17}$ In previous studies, we found that the SVVYGLR (SV) motif of the $\mathrm{N}$ fragment induced the activation of Smad signaling, leading to $\alpha$-smooth muscle actin (SMA) expression in cardiac fibroblasts. ${ }^{18,19}$ The SV peptide and OPN thrombincleaved $\mathrm{N}$ fragment increased the $\mathrm{Col} 3$ expression level in cardiac fibrosis and maintained cardiac function in a rat after ischemic cardiomyopathy (ICM) and in a dilated cardiomyopathy model hamster. ${ }^{18,20}$ However, this effect was evaluated over a short duration ( 2 weeks), and the influence of the RGD motif was not examined. In addition, the effects of increased $\mathrm{Col} 3$ expression mediated by this fragment and of the peptide on cardiac function have not yet been fully evaluated.

Accordingly, the aim of the present study was to examine the effects of $\mathrm{Col} 3$ expression on cardiac function. We transplanted fibroblast sheets that secrete $\mathrm{Col} 3$ into a model rat with ICM. Moreover, to identify the functional motif and mechanism of thrombin-cleaved $\mathrm{N}$-terminal osteopontin (N-OPN), we investigated the impact of N-OPN fragments on cardiac function and remodeling, focusing on collagen distribution.

\section{METHODS \\ Ethical Approval}

All animal work was approved by the Ethical Review Committee of Osaka University and conducted in accordance with the National Institutes of Health's (NIH) Animal Protocol Guide for the Care and Use of Laboratory Animals (NIH publication 85-23, revised 2011).

\section{Isolation and Culture of Primary Cardiac Fibroblasts}

After excision from male 3-week-old Lewis rats (Japan SLC, Shizuoka, Japan) under an overdose of sodium pentobarbital sedation $(50 \mathrm{mg} / \mathrm{kg}$, intraperitoneal injection) and heparin (150 U), the removed heart was separated from the atria, and the ventricular tissue was minced and washed with Krebs-Henseleit buffer solution. Primary adult rat ventricular fibroblasts (Fbs) were isolated using $0.2 \%$ collagenase type II (Worthington Biochemical, Lakewood, $\mathrm{NJ}$ ) and $0.1 \%$ trypsin. After centrifugation, the pelleted cells were suspended in Dulbecco's modified Eagle's medium (Nihonseiyaku, Tokyo, Japan) containing 10\% fetal bovine serum.

\section{Construction of a Human Col3-Expressing Plasmid}

A fragment of human Col3 $\alpha 1$ chain (COL3a1) cDNA including a HindIII site and secretory signal sequence at the $5^{\prime}$ end and $\mathrm{XbaI}$ sites at the $3^{\prime}$ end (Figure 1, $A$ and Figure E1) was synthesized by GenScript (Piscataway, NJ). The synthesized human COL3a1 cDNA fragment was inserted into between the HindIII and XbaI sites of the pcDNA3.1 vector.

\section{Preparation of Human COL3a1-Overexpressing Fbs}

The pcDNA3.1 expression plasmid encoding a fragment of human Col3a1 was transfected into the isolated primary Fbs using Lipofectamine (Invitrogen Life Technologies, Waltham, Mass). Stable clones expressing human Col3a1 were selected by culture under the presence of the antibiotic neomycin.

\section{Western Blot Analysis}

Cellular media were separated by sodium dodecyl sulfatepolyacrylamide gel electrophoresis. The separated proteins were transferred and incubated with anti-human $\mathrm{Col} 3$ polyclonal antibody (Proteintech Group, Chicago, Ill). The bands were visualized using an ECL kit. In contrast to the control vector cells (mock) lacking human COL3A1 cDNA, Col3 was not secreted (Figure 1, B).

\section{Transplantation of Human COL3a1-Overexpressing Fb Sheets}

To generate ICM model rats, 8-week-old male Lewis rats (Japan SLC) were anesthetized using inhaled isoflurane $(2 \% ; 0.2 \mathrm{~mL} / \mathrm{min})$ and 

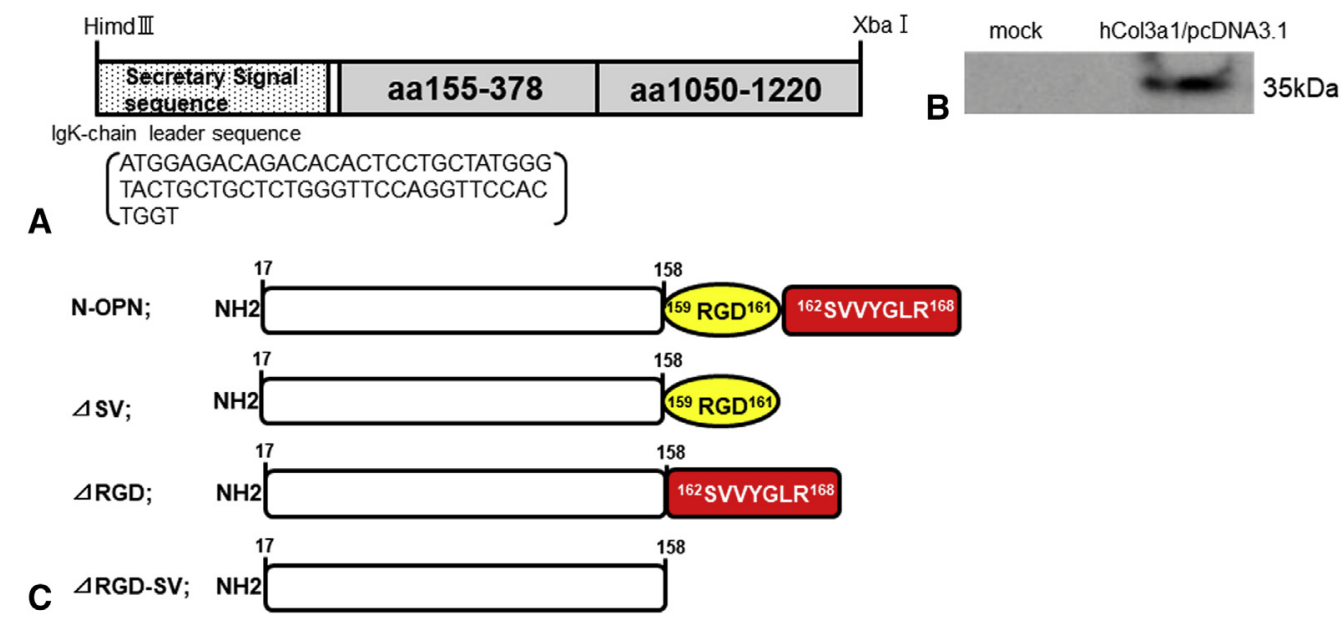

FIGURE 1. The preparation of Col3-secretory fibroblast sheets and recombinant thrombin-cleaved N-terminal osteopontin (N-OPN) fragments. A, View showing the frame format of the constructed COL3A1 gene. B, Assessment of expression and secretion of the recombinant Col3 from fibroblasts by Western blot analysis. C, Schematic view of the produced recombinant thrombin-cleaved N-OPN fragments. $R G D$, Arg-Gly-Asp.

intubated. MI was induced in the rats by ligation of the left anterior descending coronary artery (LAD). At 2 weeks after LAD ligation, Fb sheets were implanted on the infarcted LV anterior wall of the 10-week-old Lewis rats. The ICM model rats were divided into 3 treatment groups: $\mathrm{Col} 3 \mathrm{Fb}$ group (implantation of $3 \mathrm{Col} 3$-secreting $\mathrm{Fb}$ sheets; $\mathrm{n}=7$ ), (2) normal $\mathrm{Fb}$ group (implantation of 3 nontransfected $\mathrm{Fb}$ sheets; $\mathrm{n}=7$ ), and (3) control group (ligation only; $\mathrm{n}=7$ ). The 3 fibroblast sheets were sequentially applied to the surface of the infarcted myocardium. After confirming the adherence of the first sheet, subsequent sheets were applied using the same technique, as described previously. ${ }^{21-23}$

\section{Production of Human Recombinant OPN Proteins}

The coding sequences lacking the signal sequence of the thrombincleaved OPN fragments (N-OPN; amino acids 17-168), SVVYGLR sequence-removed $\mathrm{N}$-terminal OPN ( $\triangle \mathrm{SV}$; amino acids $17-161$ ), RGD sequence-removed N-terminal OPN ( $\triangle \mathrm{RGD}$; amino acids $17-158$ and 162-168), and both SVVYGLR and RGD sequence-removed N-OPN ( $\triangle$ RGD-SV; amino acids $17-158$ ) were amplified by polymerase chain reaction with specific oligonucleotides and primers and cloned into the pGEX-4T-2 vector (Figure 1, $C$ ). The expression vectors were transformed into Escherichia coli BL21, and the expressed recombinant OPN fragments were purified as described previously.

\section{Recombinant OPN Fragment or SV Peptide \\ Sustained-Release Collagen Gel Treatment}

ICM model rats were generated as described previously using 8-weekold male Sprague-Dawley rats (Charles River Laboratories Japan, Yokohama, Japan). At 2 weeks after LAD ligation, a biodegradable gelatin hydrogel (MedGel; MedGEL Corporation, Tokyo, Japan) was implanted on the LV anterior wall of the ICM model rat, as described previously. ${ }^{18,20}$ The gel incorporated a 1/10 phosphate-buffered saline (PBS) solution containing each recombinant OPN fragment, SV peptide, or random SV peptide (GYRVLSV) (each $50 \mu \mathrm{g} / \mathrm{gel}$ ). The implanted MedGel was slowly resolved by collagenase in vivo, allowing the gradual administration of physiologically active OPN fragments over a long period. A previous report confirmed that the substance integrated into the gel exhibited sustained release for 14 days after implantation in vivo. ${ }^{18}$ The rats were divided into 6 groups: N-OPN, $\triangle \mathrm{SV}, \triangle \mathrm{RGD}, \triangle \mathrm{RGD}-\mathrm{SV}, \mathrm{SV}$ peptide (SV), and random SV peptide (random). As a control, a peptide-free gel containing only PBS solution was implanted similarly $(\mathrm{n}=5$ each group).

\section{Measurement of Cardiac Function by Echocardiography}

The cardiac function of the treated rats was monitored by echocardiography both before surgery and at $2,4,6$, and 8 weeks after gel implantation or at 2 and 4 weeks after cell sheet implantation using a system equipped with a 12-MHz transducer and a SONOS 5500 device (Agilent Technologies, Santa Clara, Calif) under isoflurane inhalation $(2 \%)$, as described previously. ${ }^{19,22,23}$

\section{Histological Analysis}

At 4 or 8 weeks after treatment, the excised and fixed LV myocardium specimens were processed for histology as described previously ${ }^{22,23}$ (also see Supplemental Methods).

\section{Statistical and Power Analysis}

Data are expressed as mean $\pm \mathrm{SD}$. Differences among groups of rats at the endpoint were assessed by 1-way factorial analysis of variance; if a significant difference was detected, intergroup comparisons were performed with Tukey's honest significant difference test. Time courses of cardiac functional parameters were compared among groups by 2-way repeatedmeasures analysis of variance. A $P$ value $<.05$ (2-sided) was considered statistically significant. We performed post hoc power analysis using G*Power software.

\section{RESULTS}

\section{Effect of Col3 Expression on Cardiac Function}

No major differences at baseline were observed among the 3 experimental groups (control vs normal $\mathrm{Fb}, P=.96$; control vs $\mathrm{Col} 3 \mathrm{Fb}, P=.23$; normal $\mathrm{Fb}$ vs $\mathrm{Col} 3 \mathrm{Fb}$, $P=.79)$. The $\mathrm{Col} 3 \mathrm{Fb}$ group exhibited significant improvement in the percentage of fractional shortening $(\% \mathrm{FS})$ and ejection fraction $(\mathrm{EF})$ at 2 weeks $(P=.003)$ and 4 weeks $(P<.001)$ compared with the control group. Furthermore, at 4 weeks, significant improvements in $\% \mathrm{FS}(P=.02)$ and $\mathrm{EF}(P=.02)$ was detected between the $\mathrm{Col} 3 \mathrm{Fb}$ and normal $\mathrm{Fb}$ groups (Figure 2, $A$ and $B$ ), although the changes in percentage were small. 


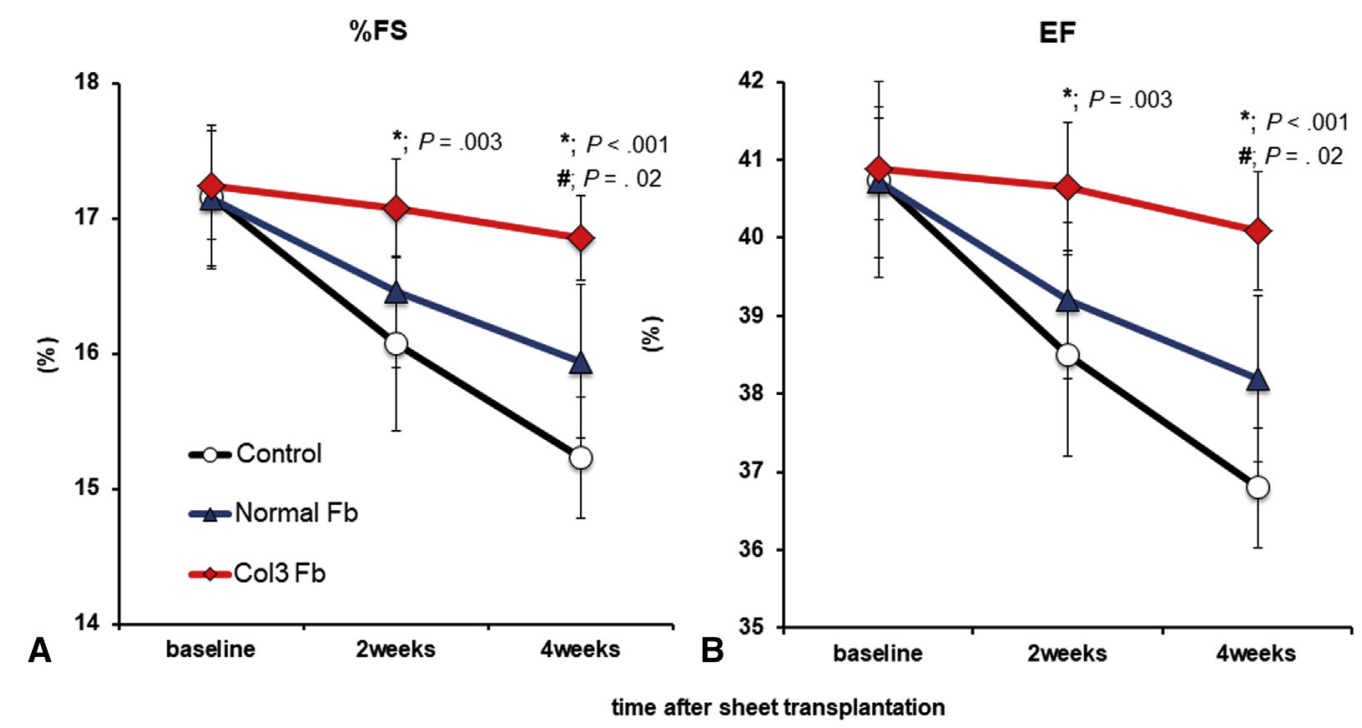

FIGURE 2. Time course of changes in left ventricular systolic function obtained by echocardiography over the 4-week period after sheet transplantation. A, Fractional shortening $(\mathrm{n}=7)$. B, Ejection fraction $(\mathrm{n}=7) . * P<.05$ versus control, $\# P<.05$ versus normal. $F S$, Fractional shortening; $F b$, fibroblast; $E F$, ejection fraction.

In contrast, there was no significant difference in both LV end-diastolic and systolic diameter (LVDd and LVDs) among the 3 experimental groups at all measurement points (Table E1).

\section{Effect of Col3 Expression on LV Remodeling}

At 4 weeks after sheet implantation, the control and normal $\mathrm{Fb}$ groups showed similar morphological changes, such as LV ventricular dilation $(P=.25)$, cardiac remodeling $(P=.45)$, and cardiomyocyte hypertrophy $(P=.25)$ (Figure $3, A-F$ ). However, the Col3 $\mathrm{Fb}$ group showed significantly attenuated $\mathrm{LV}$ ventricular dilation $(P=.03$; Figure 3, $A$ and $B$ ). Significant reductions in fibrosis (vs control, $P<.001$; vs normal $\mathrm{Fb}, P<.001$; Figure $3, C$ and $D$ ) and cardiomyocyte size (vs control, $P<.001$; vs normal $\mathrm{Fb}, P<.001$; Figure 3, $E$ and $F$ ) at infarcted border areas was observed in the $\mathrm{Col} 3 \mathrm{Fb}$ group as well.

\section{Distribution of Collagen Fiber Following Col3 Fb Sheet Implantation}

Immunohistological staining showed no significant differences in the distribution level of Col1 at infarcted areas among the 3 experimental groups (control vs normal $\mathrm{Fb}$, $P=.48$; control vs $\mathrm{Col} 3 \mathrm{Fb}, P=.65$; normal $\mathrm{Fb}$ vs $\mathrm{Col} 3$ $\mathrm{Fb}, P=.78$; Figure $3, G$ and $I$ ). Conversely, Col3 expression levels were significantly higher at the infarcted area in the $\mathrm{Col} 3 \mathrm{Fb}$ group compared with the control and normal $\mathrm{Fb}$ groups (vs control, $P<.001$; vs normal $\mathrm{Fb}, P<.001$, Figure $3, H$ and $J$ ). The ratio of Col1 to Col3 was significantly lower in the $\mathrm{Col} 3 \mathrm{Fb}$ group compared with the control and normal $\mathrm{Fb}$ groups (vs control, $P<.001$; vs normal $\mathrm{Fb}, P<.001$ : Figure $3, K$ ).

\section{Evaluation of Cardiac Function Following Gel Treatment}

Systolic function was markedly decreased over time in the control, $\triangle \mathrm{SV}, \triangle \mathrm{RGD}-\mathrm{SV}$, and random groups, whereas the N-OPN $(P<.001), \triangle$ RGD $(P<.001)$, and SV groups $(P<.001)$ exhibited significant suppression of this dysfunction at 8 weeks compared with the control group. This inhibition effect was similar among the 3 groups (EF: N-OPN vs $\triangle \mathrm{RGD}, P=.49 ; \mathrm{N}-\mathrm{OPN}$ vs $\mathrm{SV}, P=.64 ; \triangle \mathrm{RGD}$ vs $\mathrm{SV}$, $P=.22 ; \mathrm{FS}: \mathrm{N}-\mathrm{OPN}$ vs $\triangle \mathrm{RGD}, P=.68 ; \mathrm{N}-\mathrm{OPN}$ vs $\mathrm{SV}$, $P=.66 ; \Delta$ RGD vs $\mathrm{SV}, P=.37$; Figure $4, A$ and $B$ ). In contrast, both LVDd and LVDs were not significantly different among all experimental groups (Table E2).

\section{Effects of Thrombin-Cleaved OPN N-Fragment on LV Dilation and Cardiac Hypertrophy}

Histological analysis revealed that at 8 weeks after gel implantation, LV dilation (Figure 5, $A$ and $B$ ) and the $\mathrm{LV}$ cardiomyocyte cross-sectional size (Figure 5, $C$ and $D$ ) were expanded in the control, $\triangle \mathrm{SV}, \triangle \mathrm{RGD}-\mathrm{SV}$, and random groups. Conversely, these changes were significantly attenuated in the N-OPN group (LV dilation, $P=.04$; cardiomyocyte size, $P<.001$ ), $\triangle \mathrm{RGD}$ (LV dilation, $P=.005$; cardiomyocyte size, $P<.001$ ), and $\mathrm{SV}$ group (LV dilation, $P=.03$, cardiomyocyte size, $P<.001$ ) compared with the control group.

\section{Distribution of SMA and Collagen Fiber and After Gel Implantation}

The degree of myofibroblast accumulation assessed by immunostaining for $\alpha$-SMA in both the infarcted area (Figure 5,E and $F$ ) and the border area (Figure 5, $G$ 

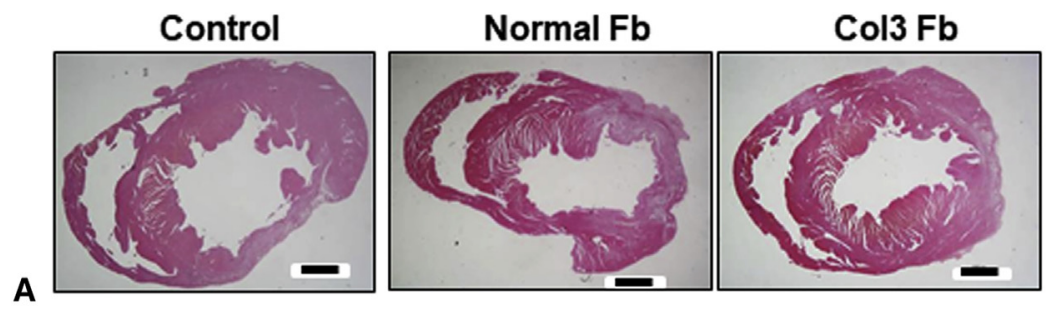

Control
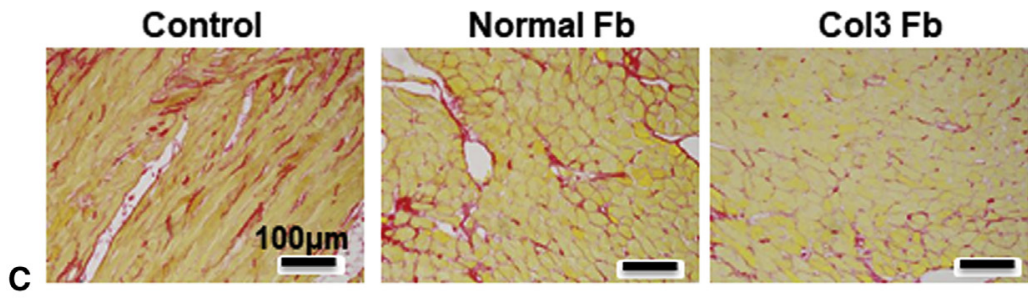

B
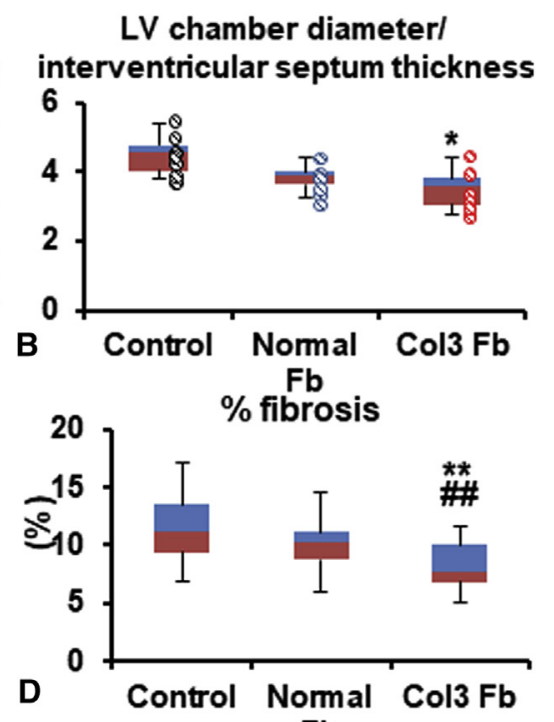

$\mathrm{Fb}$ cardiomyocyte size
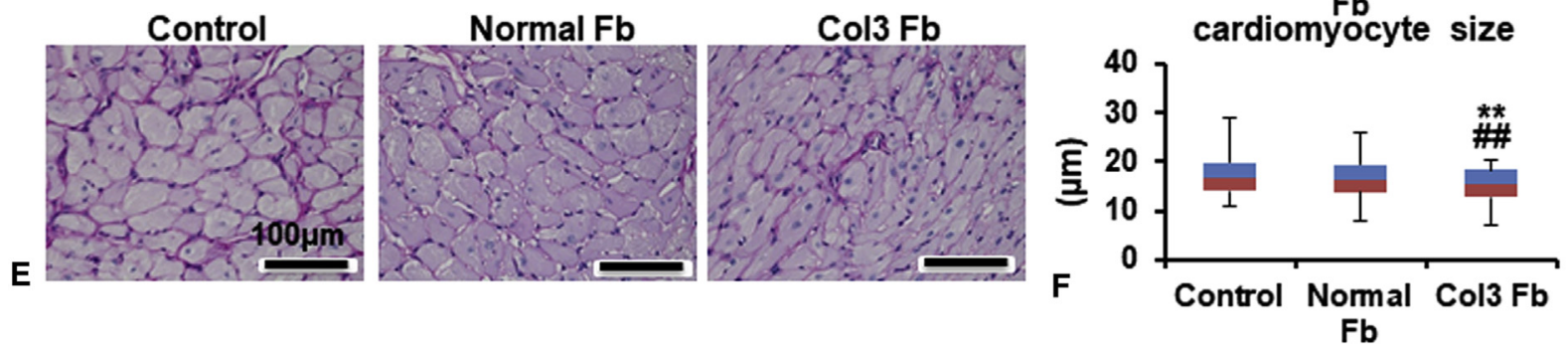

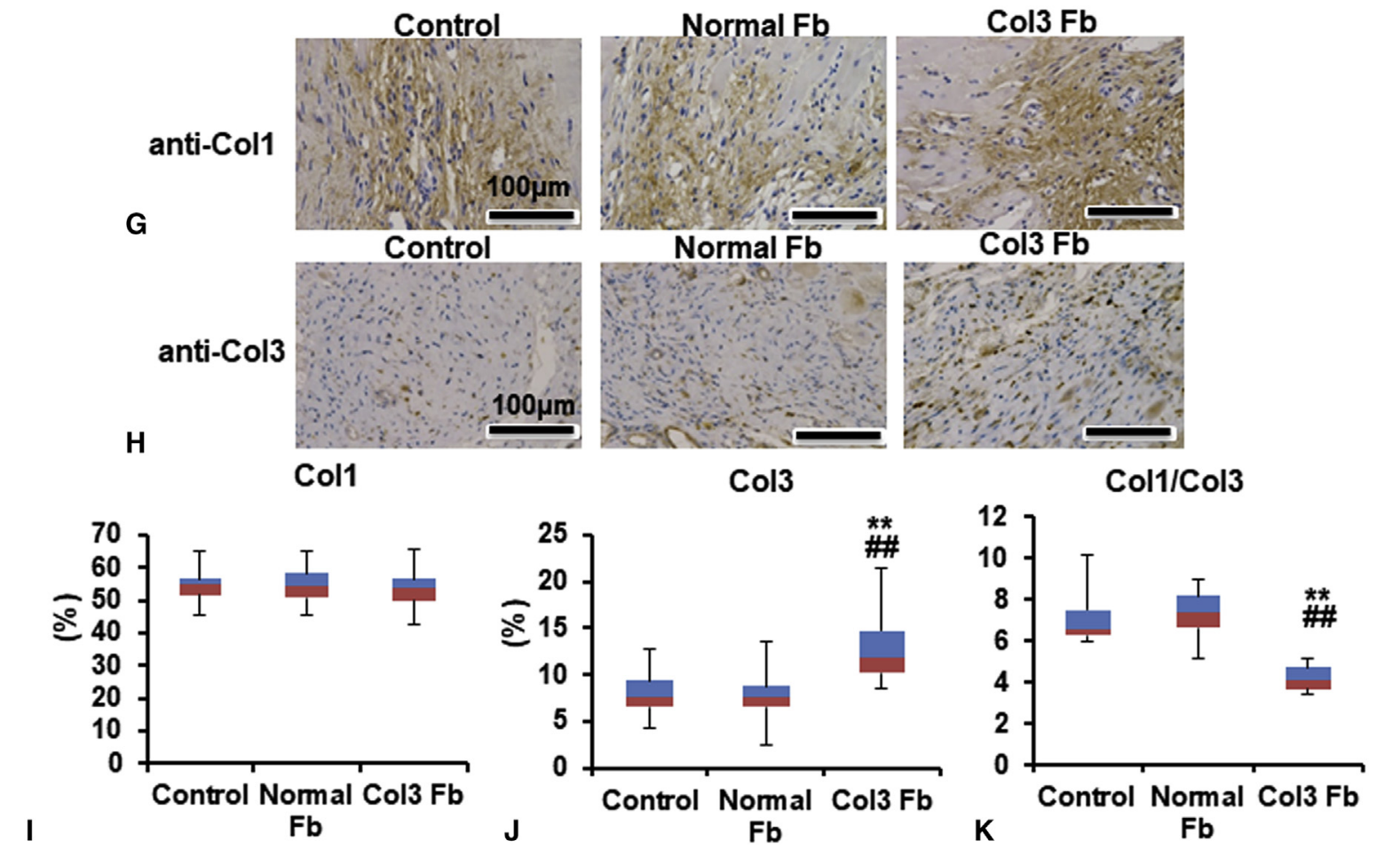

FIGURE 3. Histological evaluation of left ventricular (LV) tissue to determine LV dilation, cardiomyocyte hypertrophy, and fibrosis after 4 weeks of sheet treatment. A, Hematoxylin and eosin-stained images. (Scale bars: $1000 \mu \mathrm{m}$.) B, Ratio of LV diameter to interventricular septal thickness (n $=7$ ). C and E, Representative photomicrographs of LV cross-sections at the infarcted border area stained with Sirius red (C) and periodic acid Schiff (E). D and F, Quantification of cardiomyocyte size and fibrosis $(n=7)$. $\mathrm{G}$ and $\mathrm{H}$, Immunohistochemical analyses of 4-week rat hearts of ischemic cardiomyopathy for Col1 $(\mathrm{G})$ and Col3 $(\mathrm{H})$ at the infarcted area. I and J, Percentage of distribution of Col1 $(\mathrm{I} ; \mathrm{n}=7)$ and Col3 (J; n $=7)$. K, Ratio of Col1 to Col3 (n $=7)$. $* *<.01$, $* P<.05$ versus control; \#\#P<.01 versus normal. Fb, Fibroblast; Col3, collagen type III; $L V$, left ventricular; Coll, collagen type I. 

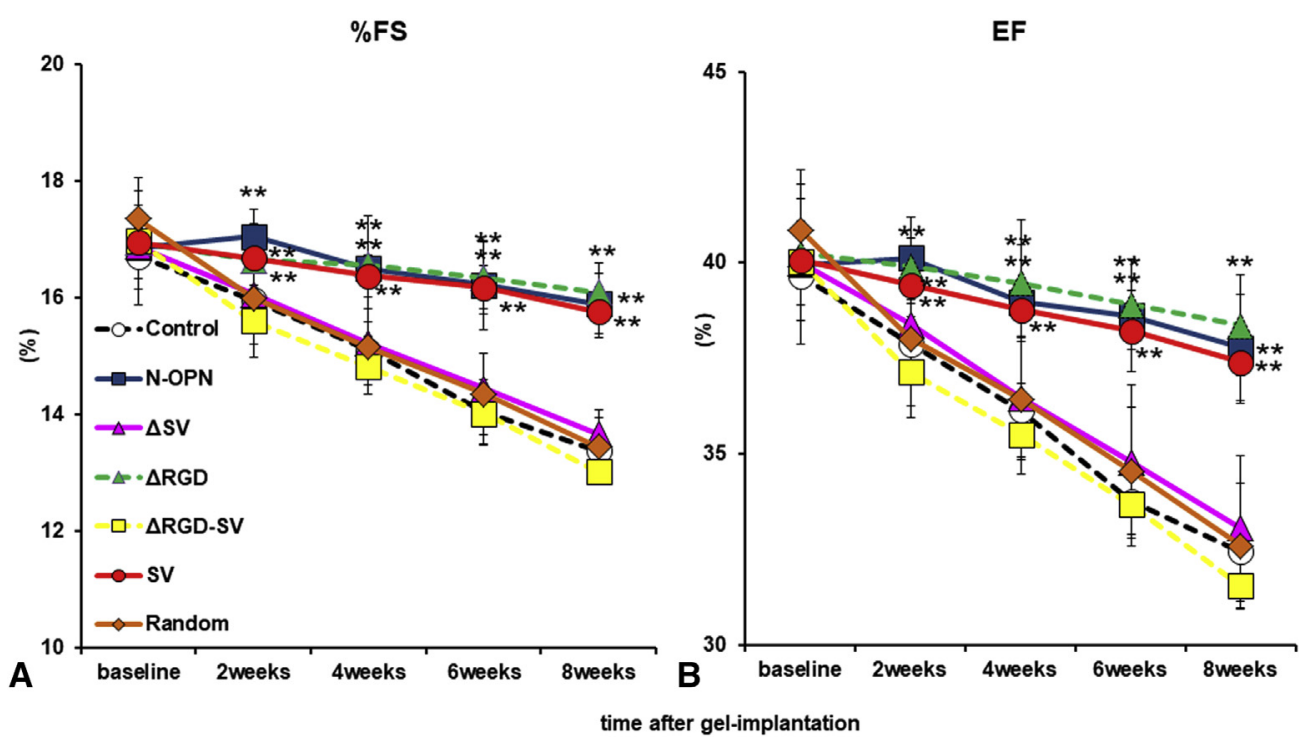

FIGURE 4. Echocardiography assessment of fractional shortening (A) and ejection fraction (B) as measured before and 2, 4, 6, and 8 weeks after gel implantation to assess left ventricular function. $\mathrm{n}=5$ in each group. ${ }^{*} P<.01$ versus control. $F S$, Fractional shortening; $N$ - $O P N$, N-terminal osteopontin; $S V$, SVVYGLR sequence; $R G D$, Arg-Gly-Asp; $E F$, ejection fraction.

and $H$ ). It was significantly higher in rats treated with recombinant N-OPN (infarcted and border areas, $P<.001$ ), $\triangle \mathrm{RGD}$ (infarcted and border areas, $P<.001$ ), and SV peptide (infarcted and border areas, $P<.001$ ) compared with the control group. The $\triangle \mathrm{SV}$ (infarcted, $P=.003$; border, $P=.03$ ) and $\triangle$ RGD-SV (infarcted; $P=.02$ ) groups also exhibited increased SMA expression in the infarcted and/or border areas, albeit to a lesser degree.

The distribution of Coll in the gel-implanted infarcted area was similar in all experimental groups $(P=.37-.52$; Figure $6, A$ and $B$ ). Conversely, the amount of $\mathrm{Col} 3$ expression in the N-OPN $(P<.001), \triangle \mathrm{RGD}(P<.001)$, and SV $(P<.001)$ groups was greater than that in the control group (Figure 6, $C$ and $D$ ). In the infarcted border area, the degree of Coll expression in the N-OPN $(P<.001), \triangle \mathrm{RGD}$ $(P<.001)$, and SV $(P<.001)$ groups was significantly decreased (Figure 6, $E$ and $F$ ), whereas that of $\mathrm{Col} 3$ in these 3 groups was increased relative to the control group (vs N-OPN, $P<.001$; vs $\triangle$ RGD; $P<.001$; vs SV, $P<.001$; Figure $6, G$ and $H$ ). Consequently, the ratio of Coll to $\mathrm{Col} 3$ in both the infarcted area (Figure $6, I$ ) and border area (Figure 6, $J$ ) was also reduced in the N-OPN (infarcted and border areas, $P<.001$ ), $\triangle$ RGD (infarcted and border areas, $P<.001$ ), and SV (infarcted and border areas, $P<.001)$ groups. The Coll distribution level in the infarcted border area, and that of $\mathrm{Col} 3$ and the Col1/Col3 ratio in both the infarcted and border areas in the $\triangle \mathrm{SV}$ (distribution of Col1, $P=.16$; distribution of Col3: infarcted, $P=.59$; border, $P=.16$; Coll/Col3 ratio: infarcted, $P=.58$; border, $P=.36$ ), $\triangle$ RGD-SV (distribution of Col1, $P=.78$; distribution of Col3: infarcted, $P=.65$; border, $P=.11$; Col1/Col3 ratio: infarcted, $P=.79$; border,
$P=.47$ ), and random (distribution of Col1, $P=.95$; distribution of Col3: infarcted, $P=.79$; border, $P=.58$; Col1/ Col3 ratio: infarcted, $P=.72$; border, $P=.66$ ) groups were comparable to those of the control group.

\section{DISCUSSION}

In this study, we have shown that (1) overexpression of Col3 attenuated cardiac systolic dysfunction by changing the balance of collagen distribution and ratio in LV remodeling; (2) the SVVYGLR motif induced Col3 expression levels in the injured myocardium; and (3) the SVVYGLR motif, but not the RGD motif, in N-OPN plays a main role in the suppression of cardiac function disruption and $\mathrm{LV}$ remodeling by increasing $\mathrm{Col} 3$ and changing the pattern of collagen balance. In the vascular structure, Coll confers rigidity, whereas $\mathrm{Col} 3$ promotes vascular elasticity. ${ }^{24}$ Specifically, higher levels of $\mathrm{Col} 3$ provide more elasticity to the ECM; for example, the stiffness of the aorta has been related to relative Col1 and Col3 abundance. ${ }^{25}$ Moreover, it has been reported that increased myocardial levels of Col1 cause unsuitable remodeling and deterioration of cardiac function owing to pressure overload, whereas increased levels of $\mathrm{Col} 3$ are associated with myocardial distensibility, leading to improved cardiac function. ${ }^{26}$ Col3 composes the initial collagen laid down on cardiac injury and is usually replaced by Col1, which consequently leads to increased stiffness of the LV wall and LV systolic dysfunction. $^{8,9,27}$ Consistent with these findings, in the present study, the implantation of Col3-secreting Fbs sheets suppressed the reduction of cardiac systolic function after ischemic damage, demonstrating that the increased level of $\mathrm{Col} 3$ distribution is an important factor in maintaining 
A
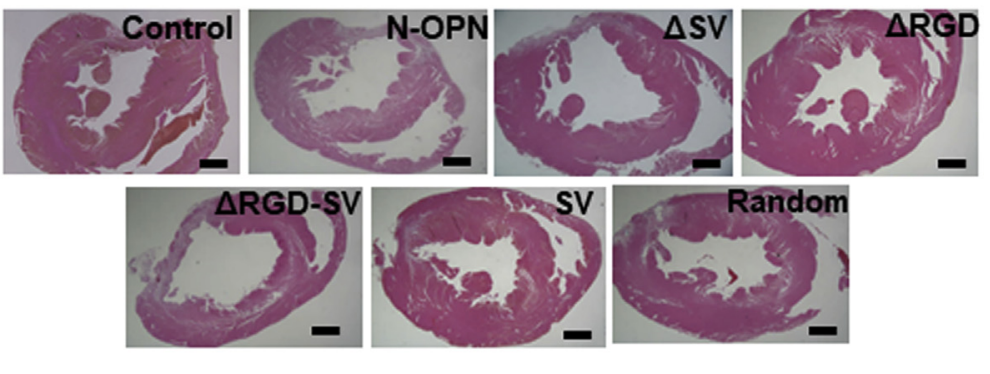

A

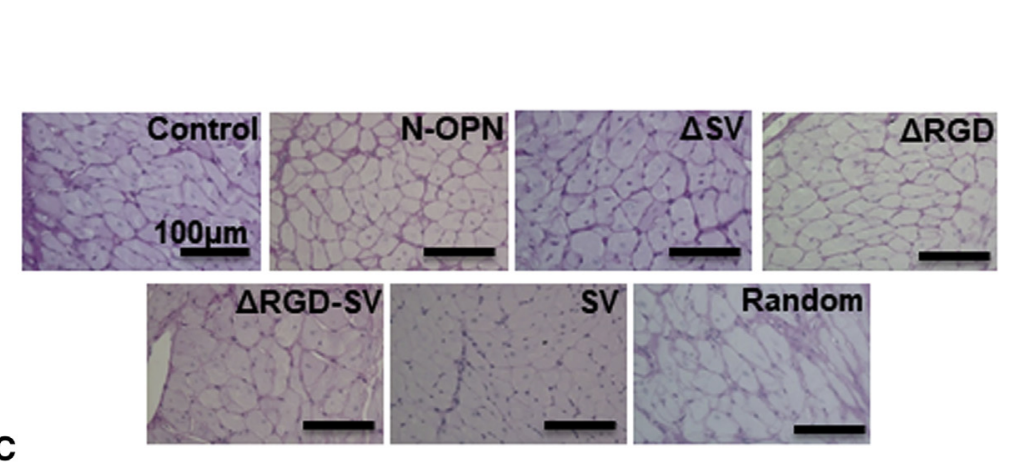

B
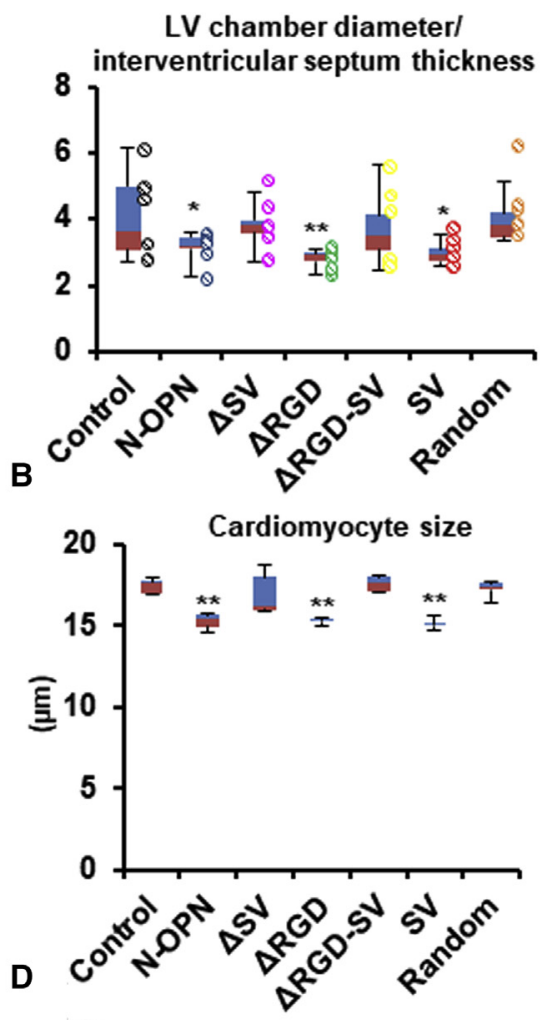

anti-SMA (infarcted area)
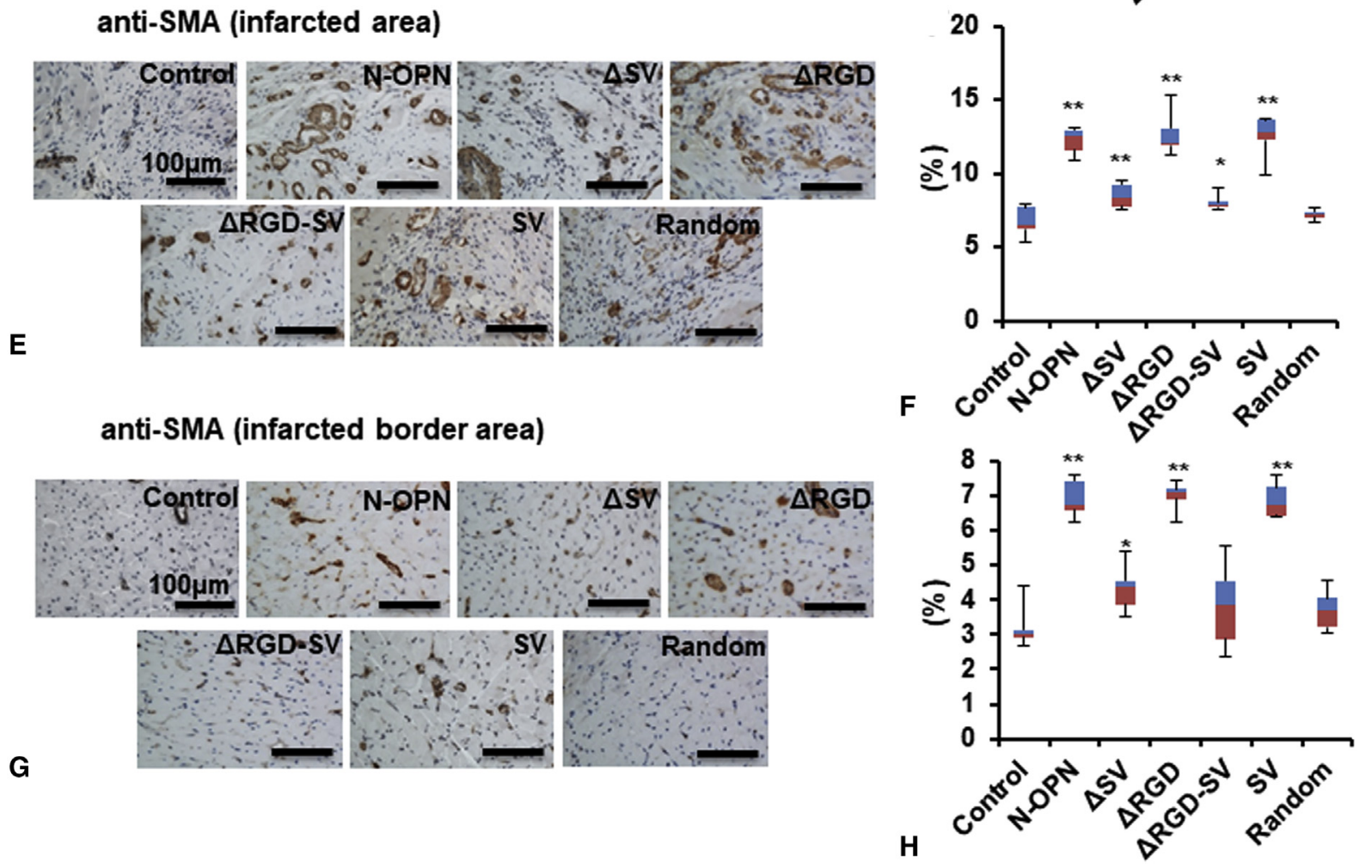

FIGURE 5. Histological changes of injured myocardium at 8 weeks after gel implantation. A, Representative image of heart sections stained with hematoxylin and eosin. (Scale bars: $1000 \mu \mathrm{m}$.) B, Quantification of the left ventricular diameter to interventricular septal thickness ratio. C, Representative images of periodic acid Schiff-stained heart sections. D, Quantification of cardiomyocyte size in the infarcted area. E and G, Immunostaining with anti- $\alpha$-SMA antibody in the infarcted (E) and border (G) areas performed on myocardium cross-sections. F and H, Percentage of SMA expression level per field area determined from sections similar to those in $(\mathrm{E})$ and $(\mathrm{G}) . \mathrm{n}=5$ in each group. ${ }^{*} * P<.01,{ }^{*} P<.05$ versus control. $N$ - $O P N$, N-terminal osteopontin; $S V$, SVVYGLR sequence; $R G D$, Arg-Gly-Asp; $L V$, left ventricular; SMA, smooth muscle actin. 

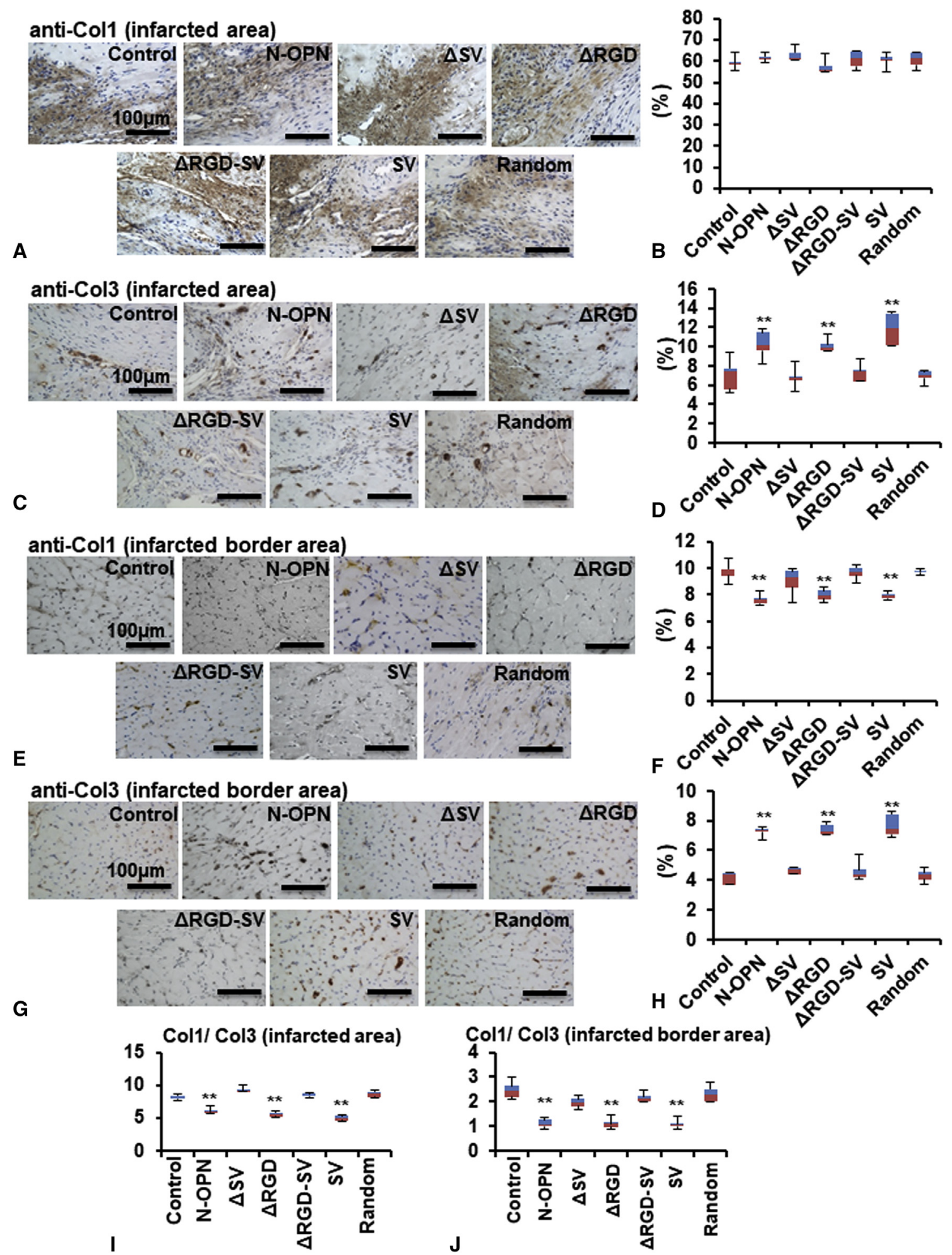

FIGURE 6. Distribution of collagen fiber in the myocardium at 8 weeks after gel implantation. A and C, Representative pictures of Col1 (A) and Col3 (C) immunohistochemical staining in the infarcted area. B and D, Percentage of sections immunopositive for Col1 (B) and Col3 (D) in the infarcted area. E and G, Representative pictures of Col1 (E) and Col3 (G) immunohistochemical staining in the infarcted area. F and $\mathrm{H}$, Percentage of sections immunopositive for Col1 $(\mathrm{F})$ and Col3 $(\mathrm{H})$ in the infarcted border area. I and J, Ratio of Col1 to Col3 in the infarcted area $(\mathrm{I})$ and the infarcted border area $(\mathrm{J})$. $\mathrm{n}=5 \mathrm{in}$ each group. $* * P<.01$ versus control. Coll, Collagen type I; $N$-OPN, N-terminal osteopontin; SV, SVVYGLR sequence; RGD, Arg-Gly-Asp; Col3, collagen type III. 
cardiac function. The differences in $\% \mathrm{FS}$ and $\mathrm{EF}$ among the groups over time are not very large; however, they indicate a significant improvement in cardiac function owing to $\mathrm{Col} 3$, and show the therapeutic effects of increasing the ratio of Col3 to Coll.

We also produced recombinant proteins corresponding to the thrombin-cleaved human N-OPN as well as N-OPN without RGD and/or SVVYGLR motifs, which were used to investigate their activity on key players in cardiac function and LV remodeling, with an emphasis on collagen distribution. Numerous studies have analyzed the distribution of OPN in various tissues, showing that it functions either as a proinflammatory cytokine or as ECM. ${ }^{12-16}$ OPN contains an RGD site that mediates its attachment to integrin and a CD44-binding domain. ${ }^{12,28,29}$ Furthermore, OPN has a thrombin cleavage site at ${ }^{168} \mathrm{RS}^{169}$ that is immediately downstream of the ${ }^{159} \mathrm{RGD}^{161} \cdot{ }^{16,17}$ Thrombin cleavage exposes a C-terminal ${ }^{162}$ SVVYGLR $^{168}$ site in the cleaved OPN that interacts with $\alpha_{9} \beta_{1}{ }^{16,17,30}$ as well as transforming growth factor (TGF) $\beta$ receptor. ${ }^{19}$ Our previous study revealed that thrombin-cleaved N-OPN and SV peptides promoted the migration of cardiac fibroblasts to the fibrotic area, where the migrated cardiac fibroblasts differentiated into myofibroblasts and produced collagen, especially $\mathrm{Col} 3$, via TGF- $\beta /$ Smad signaling in vitro and maintained cardiac function in a rat ICM model for 4 weeks. ${ }^{18}$ However, our previous studies did not include a detailed evaluation of the $\mathrm{N}$-OPN motif(s) important for these effects or the impact of increased $\mathrm{Col} 3$ expression on cardiac function mediated by this fragment in vivo.

The RGD sequence is present in many ECM proteins and can interact with integrin at focal adhesion points. ${ }^{12,31}$ This interaction activates signal transduction between the cell and the ECM, thereby influencing cell motility, including proliferation, differentiation, migration, and survival. However, in the present study, the fragment containing the SVVYGLR motif and SV peptide significantly inhibited cardiac depression and increased $\mathrm{Col} 3$ expression in impaired myocardium, whereas the $\triangle \mathrm{SV}$ fragment had no effect on these changes. This implies that the SVVVYGLR motif, rather than the RGD motif, played a key function in the effects of N-OPN on cardiac function and LV remodeling. Furthermore, the N-OPN fragments containing the SVVYGLR motif and SV peptide caused the increase of $\mathrm{Col} 3$ expression and altered the ratio of Col1 to Col3. These results also demonstrate that this change in collagen distribution and the reduced ratio of Col1 to Col3 likely have crucial roles in the attenuation of cardiac dysfunction mediated by these fragments.

Both Col1 and Col3 have been shown to be closely associated with myocardial fibrosis, and their levels are increased in the LV remodeling process. ${ }^{32}$ The contractility of the myocardium is determined by the viscoelasticity and relative content of Coll and Col3. ${ }^{32}$ The degree of cross- linking and the ratio of newly synthesized Col1 and Col3 have important roles in cardiac function. Thus, our study evaluated the ratios among collagen types, and found that cardiac function after cell sheet or gel implantation was maintained in rats with ICM.

TGF- $\beta 1 /$ Smad signaling induces the expression of the $\alpha$-SMA gene, a well-known marker of myofibroblast differentiation. ${ }^{33}$ The SVVYGLR motif and SV peptide can induce the differentiation of cardiac fibroblasts to myofibroblasts through binding of the TGF- $\beta$ receptor. ${ }^{18,19}$ According to our previous studies, as the fragment containing the SVVYGLR motif and SV peptide increased SMA expression in the present study, this suggests that the SVVYGLR/Smad signal transduction via the TGF- $\beta$ receptor induced a beneficial effect on cardiac function by increasing SMA-positive myofibroblast-like cells. Myofibroblasts constitute a major source of ECM proteins, especially collagen. ${ }^{33}$ The accumulation of myofibroblasts and the changes in the amount and/or distribution of collagen by myofibroblasts accordingly affect heart function. ${ }^{33,34}$ Consistent with previous reports, in the present study, the accumulated myofibroblasts stimulated by SVVYGLR motif and SV peptide led to changes in collagen distribution. Furthermore, the N-OPN fragments lacking the SVVYGLR motif also caused increased expression of SMA, although the expression level was lower than in the N-OPN, $\triangle \mathrm{RGD}$, and random groups. Therefore, a functional site other than the SVVYGLR and RGD motifs is likely involved in the induction of SMA expression.

This study had some limitations. We found it difficult to express full-length Col3a1 by gene transfer to primary $\mathrm{Fbs}$, and instead used a fragmented Col3a1, which has the same structural quality as the full-length protein. As reported previously, this fragmented Col3al contains the repeated Gly-X-Y structural motif that is necessary for perfect triple-helix formation, and is equivalent to full-length Col3 in terms of hydroxylation efficiency, proline and lysine residue distribution, and triple-helix conformation thermal stability. ${ }^{35,36}$ In addition, owing to the relatively small sample size, the individual variation within the same group was considerable. Statistical analysis showed a significant difference between Col3 or SVVYGLR motif treatment and control groups; however, the power analysis results suggest that the sample size was too small to provide sufficient statistical power to detect a significant difference, and that a relatively large sample size is needed to claim a statistical difference. Data from a larger sample could reflect the effects of $\mathrm{Col} 3$ and the SVVYGLR motif on cardiac remodeling and function.

\section{CONCLUSIONS}

In conclusion, in our series, the increased distribution of $\mathrm{Col} 3$ in injured myocardium prevented cardiac dysfunction 
by changing the balance of collagen distribution and ratio in LV remodeling. The SVVYGLR motif in the thrombincleaved OPN has a central role in the maintenance of cardiac function, and $\mathrm{Col} 3$ expression plays an important role in the beneficial effects on cardiac function mediated by the OPN fragment containing SVVYGLR and SV peptides in ICM. Some previous studies have reported the biomechanical effects of Col3 in pulmonary abnormalities and urinary bladder filling. ${ }^{37,38}$ Based on the effects of Col3 in this study, we consider that the elasticity of Col3 can be applied to therapeutic cardiac biomechanics.

\section{Conflict of Interest Statement}

Authors have nothing to disclose with regard to commercial support.

\section{References}

1. Colucci WS. Molecular and cellular mechanisms of myocardial failure. Am J Cardiol. 1997;80:15L-25L.

2. Weber KT, Brilla CG. Pathological hypertrophy and cardiac interstitium: fibrosis and renin-angiotensin-aldosterone system. Circulation. 1991;83:1849-65.

3. Davies CH, Davia K, Bennett JG, Pepper JR, Poole-Wilson PA, Harding SE Reduced contraction and altered frequency response of isolated ventricular myocytes from patients with heart failure. Circulation. 1995;92:2540-9.

4. Borg TK, Caulfield JB. The collagen matrix of the heart. Fed Proc. 1981;40: 2037-41.

5. Weber KT. Cardiac interstitium in health and disease: the fibrillar collagen network. J Am Coll Cardiol. 1989;13:1637-52.

6. Yoshikane H, Honda M, Goto Y, Morioka S, Ooshima A, Moriyama K. Collagen in dilated cardiomyopathy: scanning electron microscopic and immunohistochemical observations. Jpn Circ J. 1992:56:899-910.

7. Lapiere CM, Nusgens B, Pierard GE. Interaction between collagen type I and type III in conditioning bundles organization. Connect Tissue Res. 1977;5:21-9.

8. Pauschinger M, Knopf D, Petschauer S, Doerner A, Poller W, Schwimmbeck PL, et al. Dilated cardiomyopathy is associated with significant changes in collagen type I/III ratio. Circulation. 1999;99:2750-6.

9. Kitamura M, Shimizu M, Ino H, Okeie K, Yamaguchi M, Funjno N, et al Collagen remodeling and cardiac dysfunction in patients with hypertrophic cardiomyopathy: the significance of type III and VI collagens. Clin Cardiol. 2001; 24:325-9.

10. Marijianowski MM, Teeling P, Mann J, Becker AE. Dilated cardiomyopathy is associated with an increase in the type I/type III collagen ratio: a quantitative assessment. J Am Coll Cardiol. 1995;25:1263-72.

11. Brodsky B, Persikov AV. Molecular structure of the collagen triple helix. Adv Protein Chem. 2005;70:301-39.

12. Liaw L, Lindner V, Schwartz SM, Chambers AF, Giachelli CM. Osteopontin and beta 3 integrin are coordinately expressed in regenerating endothelium in vivo and stimulate Arg-Gly-Asp-dependent endothelial migration in vitro. Cric Res. 1995;77:665-72.

13. El-Tanani MK, Campbell FC, Kurisetty V, Jin D, McCann M, Rudland PS. The regulation and role of osteopontin in malignant transformation and cancer. Cytokine Growth Factor Rev. 2006;17:463-74.

14. Lund SA, Giachelli CM, Scatena M. The role of osteopontin in inflammatory processes. J Cell Commun Signal. 2009;3:311-22.

15. Liaw L, Almeida M, Hart CE, Schwartz SM, Giachelli CM. Osteopontin promotes vascular cell adhesion and spreading and is chemotactic for smooth muscle cells in vitro. Circ Res. 1994;74:214-24.

16. Smith LL, Giachelli CM. Structural requirements for $\alpha 9 \beta 1$-mediated adhesion and migration to thrombin-cleaved osteopontin. Exp Cell Res. 1998;242:351-60.

17. Green PM, Ludbrook SB, Miller DD, Horgan CM, Barry ST. Structural elements of the osteopontin SVVYGLR motif important for the interaction with $\alpha(4)$ integrins. FEBS Lett. 2001;503:75-9.

18. Uchinaka A, Hamada Y, Mori S, Miyagawa S, Saito A, Sawa Y, et al. SVVYGLR motif of the thrombin-cleaved N-terminal osteopontin fragment enhances the synthesis of collagen type III in myocardial fibrosis. Mol Cell Biochem. 2015 408:191-203.

19. Uchinaka A, Kawaguchi N, Hamada Y, Mori S, Miyagawa S, Saito A, et al. Transplantation of myoblast sheets that secrete the novel peptide SVVYGLR improves cardiac function in failing hearts. Cardiovasc Res. 2013;99:102-10.

20. Mizuno Y, Uchinaka A, Horii Y, Mori S, Hamada Y, Miyagawa S, et al. Improvement of cardiac function after implanting the osteopontin-derived peptide SVVYGLR in a hamster model of dilated cardiomyopathy. Interact Cardiovas Thorac Surg. 2015;21:506-14.

21. Sekiya N, Matsumiya G, Miyagawa S, Saito A, Shimizu T, Okano T, et al. Layered implantation of myoblast sheets attenuates adverse cardiac remodeling of the infarcted heart. J Thorac Cardiovasc Surg. 2009;138:985-93.

22. Uchinaka A, Kawaguchi N, Hamada Y, Miyagawa S, Saito A, Mori S, et al Transplantation of elastin-secreting myoblast sheets improves cardiac function in infarcted rat heart. Mol Cell Biochem. 2012;368:203-14.

23. Uchinaka A, Tasaka K, Mizuno Y, Maeno Y, Ban T, Mori S, et al. Laminin $\alpha 2$ secreting fibroblasts enhance the therapeutic effect of skeletal myoblast sheets Eur J Cardiothorac Surg. 2017;51:457-64.

24. Pfeffer MA, Braunwald E. Ventricular remodeling after myocardial infarction experimental observations and clinical implications. Circulation. 1990;81: 1161-72.

25. Qiu H, Depre C, Ghosh K, Resuello RG, Natividad FF, Rossi F, et al. Mechanism of gender-specific differences in aortic stiffness with aging in nonhuman primates. Circulation. 2007;116:669-76.

26. Ravichandran LV, Puvanakrishnan R. In vivo labeling studies on the biosynthesis and degradation of collagen in experimental myocardial infarction. Biochem Int. 1991:24:405-14.

27. Weber KT, Jalil JE, Janicki JS, Pick R. Myocardial collagen remodeling in pressure overload hypertrophy: a case for interstitial heart disease. Am J Hypertens. 1989;2(12 Pt 1):931-40.

28. Maeda N, Ohashi T, Chagan-Yasutan H, Hattori T, Takahashi Y, Harigae H, et al. Osteopontin-integrin interaction as a novel molecular target for antibody-mediated immunotherapy in adult T-cell leukemia. Retrovirology. 2015;12:99.

29. Ohashi R, Tajima K, Takahashi F, Cui R, Gu T, Shimizu K, et al, Osteopontin modulates malignant pleural mesothelioma cell functions in vitro. Anticancer Res. 2009;29:2205-14

30. Yokosaki Y, Matsuura N, Sasaki T, Murakami I, Schneider H, Higashiyama S, et al. The integrin alpha(9)beta(1) binds to a novel recognition sequence (SVVYGLR) in the thrombin-cleaved amino-terminal fragment of osteopontin. J Biol Chem. 1999;274:36328-34.

31. Cavalcanti-Adam EA, Volberg T, Micoulet A, Kessler H, Geiger B, Spatz JP. Cell spreading and focal adhesion dynamics are regulated by spacing of integrin ligands. Biophys J. 2007:92:2964-74.

32. Weber KT, Janicki JS, Shroff SG, Pick R, Chen RM, Bashey RI. Collagen remodeling of the pressure-overloaded, hypertrophied nonhuman primate myocardium. Circ Res. 1988;62:757-65.

33. Vaughan MB, Howard EW, Tomasek JJ. Transforming growth factor-beta1 promotes the morphological and functional differentiation of the myofibroblast. Exp Cell Res. 2000;257:180-9.

34. Dobaczewski M, Bujak M, Li N, Gonzalez-Quesada C, Mendoza LH, Wang XF, et al. Smad3 signaling critically regulates fibroblast phenotype and function in healing myocardial infarction. Circ Res. 2010;107:418-28.

35. Rutschmann C, Baumann S, Cabalzar J, Luther KB, Hennet T. Recombinant expression of hydroxylated human collagen in Escherichia coli. Appl Microbiol Biotechnol. 2014;98:4445-55.

36. Ramshaw JAM, Shah NK, Brodsky B. Gly-X-Y tripeptide frequencies in collagen: a context for host-guest triple-helical peptides. J Struct Biol. 1998; 122:86-91.

37. Suki B, Ito S, Stamenovic D, Lutchen KR, Ingenito EP. Biomechanics of the lung parenchyma: critical roles of collagen and mechanical forces. J Appl Physiol (1985). 2005;98:1892-9.

38. Chang SL, Howard PS, Koo HP, Macarak EJ. Role of type III collagen in bladder filling. Neurourol Urodyn. 1998;17:135-45.

Key Words: collagen type III, remodeling, ischemic cardiomyopathy 


\section{APPENDIX. SUPPLEMENTAL METHODS Isolation and Culture of Primary Cardiac Fibroblasts}

Primary adult rat ventricular fibroblasts (Fbs) were incubated with $0.2 \%$ collagenase type II (Worthington Biochemical, Lakewood, NJ) and $0.1 \%$ trypsin at $37^{\circ} \mathrm{C}$ for 10 minutes under constant shaking. Isolated cells were pelleted at the end of each 10-minute digestion period by centrifugation. Cells from the second to ninth digestions were plated on 100-mm culture dishes in Dulbecco's modified Eagle's medium (Nihonseiyaku, Tokyo, Japan) containing $10 \%$ fetal bovine serum. The isolated cells were immunofluorescently stained with anti-fibronectin antibody (DAKO, Carpinteria, Calif) to confirm the density or uniqueness of fibroblasts from the suspended cell pellet. The density of fibroblast was roughly $80 \%-90 \%$.

\section{Cell Sheet Preparation}

The primary Fbs at a density of $3 \times 10^{6}$ cells/dish were seeded in a temperature-responsive culture dish (Upcell; CellSeed, Tokyo, Japan) and incubated until a confluent culture was obtained at $37^{\circ} \mathrm{C}$. The cell sheet was harvested by lowering the culture temperature to $20^{\circ} \mathrm{C}$.

\section{Transplantation of Fb Sheets and Collagen Gel}

We opened the cardiac membrane and placed the sheets over the scared area without the use of suture. With a temperature-responsive cell culture dish, cell sheets consisting of several million cells can be readily manufactured with no added scaffold material. These cell sheets maintain cell-cell and cell-ECM adhesion, because the proteases like trypsin are not used when they are collected. Thus, the implanted sheets adhere to myocardium immediately and do not fall off after chest closure. Three fibroblast sheets were applied sequentially to the surface of the infarcted myocardium. Once adherence of the first sheet was confirmed, subsequent sheets were applied using the same technique. The implanted sheets were stacked on the infarcted myocardium.

The recombinant OPN or SV peptide-impregnated gels were transplanted onto the LV anterior wall of a rat with MI, covering the whole infarcted anterior wall. Implanted gels were approximately $1 \mathrm{~cm}^{2}$. The pericardium was overwrapped by suturing with 8-0 Prolene polypropylene suture (Ethicon, Somerville, NJ) to fix to the epicardial surface. Because the infarcted area showed macroscopically whitish myocardium, we implanted sheets or gel onto the area to not contact with the border zone or remote LV tissue.

\section{Histological Analysis}

The extent of LV dilation was calculated as LV chamber diameter/interventricular septal thickness. The percentage of fibrosis was quantitatively analyzed by point counting. Cardiomyocyte hypertrophy was evaluated by measuring the 2-point shortest axes at the level of the nucleus. Immunohistochemical staining with antibodies against Col1 (Col1; Abcam, Cambridge, UK) and Col3 (monoclonal anti-Col3 antibody for analysis of gel implanation; Novus Biologicals, Littleton, Colo or polyclonal anti-Col3 antibody for analysis of cell sheet implantation; Proteintech Group, Rosemont, Ill) was also performed to assess of the level of collagen expression. The distribution of collagen was described as Col1- or Col3-positive area/field of view $\times 100(\%)$. The sections were incubated with monoclonal mouse anti- $\alpha$-SMA (DAKO) to detect myofibroblast accumulation. All image analyses were performed using Scion Image software (National Institutes of Health, Frederick, Md). 
MMSFVQKGSWLLLALLHPTIILAQQEAVEGGCSHLGQSYADRDV

WKPEPCQICVCDSGSVLCDDICDDQELDCPNPEIPFGECCAVCPQPPTAPTRPPNGQ

GPQGPKGDPGPPGIPGRNGDPGIPGQPGSPGSPGPPGICESCPTGPQNYSPQYDSYDV

KSGVAVGGLAGYPGPAGPPGPPGPPGTSGHPGSPGSPGYQGPPGEPGQAGPSGPPGPP GAIGPSGPAGKDGESGRPGRPGERGLPGPPGIKGPAGIPGFPGMKGHRGFDGRNGEKG ETGAPGLKGENGLPGENGAPGPMGPRGAPGERGRPGLPGAAGARGNDGARGSDGQPGP PGPPGTAGFPGSPGAKGEVGPAGSPGSNGAPGQRGEPGPQGHAGAQGPPGPPGINGSP GGKGEMGPAGIPGAPGLMGARGPPGPAGANGAPGLRGGAGEPGKNGAKGEPGPRGERG EAGIPGVPGAKGEDGKDGSPGEPGANGLPGAAGERGAPGFRGPAGPNGIPGEKGPAGE RGAPGPAGPRGAAGEPGRDGVPGGPGMRGMPGSPGGPGSDGKPGPPGSQGESGRPGPP GPSGPRGQPGVMGFPGPKGNDGAPGKNGERGGPGGPGPQGPPGKNGETGPQGPPGPTG PGGDKGDTGPPGPQGLQGLPGTGGPPGENGKPGEPGPKGDAGAPGAPGGKGDAGAPGE RGPPGLAGAPGLRGGAGPPGPEGGKGAAGPPGPPGAAGTPGLQGMPGERGGLGSPGPK GDKGEPGGPGADGVPGKDGPRGPTGPIGPPGPAGQPGDKGEGGAPGLPGIAGPRGSPG ERGETGPPGPAGFPGAPGQNGEPGGKGERGAPGEKGEGGPPGVAGPPGGSGPAGPPGP QGVKGERGSPGGPGAAGFPGARGLPGPPGSNGNPGPPGPSGSPGKDGPPGPAGNTGAP GSPGVSGPKGDAGQPGEKGSPGAQGPPGAPGPLGIAGITGARGLAGPPGMPGPRGSPG PQGVKGESGKPGANGLSGERGPPGPQGLPGLAGTAGEPGRDGNPGSDGLPGRDGSPGG KGDRGENGSPGAPGAPGHPGPPGPVGPAGKSGDRGESGPAGPAGAPGPAGSRGAPGPQ GPRGDKGETGERGAAGIKGHRGFPGNPGAPGSPGPAGQQGAIGSPGPAGPRGPVGPSG PPGKDGTSGHPGPIGPPGPRGNRGERGSEGSPGHPGQPGPPGPPGAPGPCCGGVGAAA IAGIGGEKAGGFAPYYGDEPMDFKINTDEIMTSLKSVNGQIESLISPDGSRKNPARNC RDLKFCHPELKSGEYWVDPNQGCKLDAIKVFCNMETGETCISANPLNVPRKHWWTDSS AEKKHVWFGESMDGGFQFSYGNPELPEDVLDVQLAFLRLLSSRASQNITYHCKNSIAY MDQASGNVKKALKLMGSNEGEFKAEGNSKFTYTVLEDGCTKHTGEWSKTVFEYRTRKA VRLPIVDIAPYDIGGPDQEFGVDVGPVCFL

aa155-378

aa1050-1220

FIGURE E1. DNA sequence of the synthetic human Col3 $\alpha 1$ chain (COL3A1) DNA construct.

TABLE E1. Echocardiographic assessment of LVDd and LVDs over time by after sheet implantation

\begin{tabular}{lccc}
\hline Parameter & Baseline & $\mathbf{2 ~ W k}$ & $\mathbf{4}$ Wk \\
\hline LVDd, mm & & & \\
$\quad$ Control & $0.739 \pm 0.107$ & $0.749 \pm 0.112$ & $0.753 \pm 0.118$ \\
Normal Fb & $0.756 \pm 0.094$ & $0.756 \pm 0.099$ & $0.746 \pm 0.125$ \\
Col3 Fb & $0.724 \pm 0.011$ & $0.716 \pm 0.092$ & $28.68 \pm 1.66^{*}, \dagger$ \\
LVDs, mm & & & \\
Control & $0.593 \pm 0.089$ & $0.620 \pm 0.087$ & $0.644 \pm 0.097$ \\
Normal Fb & $0.620 \pm 0.068$ & $0.663 \pm 0.111$ & $0.661 \pm 0.128$ \\
Col3 Fb & $0.599 \pm 0.092$ & $0.593 \pm 0.075$ & $0.611 \pm 0.106$ \\
\hline
\end{tabular}

Baseline is the time of transplantation, which was 2 weeks after ligation of LAD. Other times are weeks post-transplantation. $L V D d$, Left ventricular end-diastolic dimension; $\mathrm{Fb}$, fibroblast; $\mathrm{Col} 3$, collagen type III; $L V D s$, left ventricular end-systolic dimension. $* P<.05$ versus control. $\dagger P<.05$ versus normal $\mathrm{Fb}$. 
TABLE E2. Echocardiographic assessment of LVDd and LVDs over time after gel implantation

\begin{tabular}{lcccrr}
\hline Parameter & Baseline & $\mathbf{2 ~ W k}$ & $\mathbf{4 ~ W k}$ & $\mathbf{6 ~ W k}$ & $\mathbf{8 ~ W k}$ \\
\hline LVDd, mm & & & & & \\
Control & $0.860 \pm 0.149$ & $0.895 \pm 0.184$ & $0.876 \pm 0.113$ & $0.978 \pm 0.142$ & $0.969 \pm 0.228$ \\
N-OPN & $0.789 \pm 0.052$ & $0.917 \pm 0.131$ & $0.957 \pm 0.161$ & $0.896 \pm 0.101$ & $0.964 \pm 0.142$ \\
$\triangle$ RGD & $0.822 \pm 0.092$ & $0.888 \pm 0.128$ & $0.889 \pm 0.122$ & $0.939 \pm 0.104$ & $0.943 \pm 0.151$ \\
$\triangle$ SV & $0.791 \pm 0.081$ & $0.802 \pm 0.157$ & $0.901 \pm 0.148$ & $0.898 \pm 0.104$ & $0.831 \pm 0.178$ \\
$\triangle$ RGD-SV & $0.889 \pm 0.100$ & $0.898 \pm 0.095$ & $0.932 \pm 0.091$ & $0.944 \pm 0.103$ & $0.949 \pm 0.161$ \\
SV & $0.858 \pm 0.185$ & $0.905 \pm 0.099$ & $0.949 \pm 0.106$ & $0.983 \pm 0.170$ & $0.965 \pm 0.166$ \\
Random & $0.828 \pm 0.099$ & $0.926 \pm 0.096$ & $0.908 \pm 0.141$ & $0.937 \pm 0.115$ & $0.923 \pm 0.157$ \\
LVDs, mm & & & & $0.845 \pm 0.119$ & \\
Control & $0.717 \pm 0.126$ & $0.753 \pm 0.158$ & $0.773 \pm 0.119$ & $0.840 \pm 0.196$ \\
N-OPN & $0.658 \pm 0.050$ & $0.778 \pm 0.108$ & $0.799 \pm 0.142$ & $0.748 \pm 0.084$ & $0.815 \pm 0.127$ \\
$\triangle$ RGD & $0.700 \pm 0.048$ & $0.749 \pm 0.107$ & $0.756 \pm 0.105$ & $0.820 \pm 0.110$ & $0.805 \pm 0.140$ \\
$\triangle$ SV & $0.658 \pm 0.068$ & $0.668 \pm 0.133$ & $0.737 \pm 0.132$ & $0.751 \pm 0.123$ & $0.699 \pm 0.154$ \\
$\triangle$ RGD-SV & $0.738 \pm 0.082$ & $0.758 \pm 0.081$ & $0.794 \pm 0.078$ & $0.809 \pm 0.086$ & $0.828 \pm 0.161$ \\
SV & $0.713 \pm 0.159$ & $0.756 \pm 0.106$ & $0.814 \pm 0.118$ & $0.824 \pm 0.143$ & $0.814 \pm 0.143$ \\
Random & $0.694 \pm 0.084$ & $0.776 \pm 0.087$ & $0.779 \pm 0.118$ & $0.803 \pm 0.108$ & $0.783 \pm 0.143$ \\
\hline
\end{tabular}

Baseline is time of transplantation, which was 2 weeks after ligation of LAD. Other times in weeks are post-transplantation. $L V D d$, Left ventricular end-diastolic dimension; $N$-OPN, N-terminal osteopontin; $R G D$, Arg-Gly-Asp; SV, SVVYGLR sequence; $L V D s$, left ventricular end-systolic dimension. 